\title{
El liderazgo como factor clave para el mejoramiento de la profesionalización docente y la calidad educativa
}

\author{
Claudia Patricia Baloco Navarro \\ claudiabaloco@mail.uniatlantico.edu.co \\ Universidad del Atlántico
}

\section{Resumen}

En este documento se abordan algunos de los temas coyunturales de la profesionalización docente a la luz de las políticas públicas en Colombia y la relación del liderazgo como factor clave para el mejoramiento de la calidad educativa. Se toca además la pertinencia de las recomendaciones de la OECD en cuanto a la formación docente para Colombia, por lo cual es importante realizar una exploración crítica de los dispositivos de formación docente a la luz de las políticas de perfeccionamiento, para avanzar en el análisis y desarrollo de propuestas alternativas y nuevas orientaciones para el desarrollo profesional docente. Para el desarrollo de esta propuesta ha sido necesario generar una acción reflexiva sistemática y crítica acerca de la relación del liderazgo como factor clave para lograr calidad educativa, a la luz de las políticas públicas. Al revisar la normatividad de la profesión docente en Colombia, se concluye que hay aspectos faltantes para lograr que el docente sea realmente un líder proactivo y empático. Factores claves para la formación docente y el mejoramiento de la calidad educativa en el proceso de pensar en configurar las escuelas como comunidades profesionales de aprendizaje.

\section{Palabras clave}

Políticas educativas; Liderazgo; profesionalización docente; calidad educativa; 


\title{
Leadership as a key factor for improving teacher professionalization and educational quality
}

\begin{abstract}
This document addresses some of the conjunctural issues of teacher professionalization in light of public policy in Colombia and the relationship of leadership as a key factor in improving educational quality. The relevance of the OECD recommendations regarding teacher training for Colombia is also touched on, so it is important to carry out a critical exploration of teacher training devices in light of the improvement policies, to advance in the analysis and development of alternative proposals and new guidelines for teacher professional development. For the development of this proposal it has been necessary to generate a systematic and critical reflective action about the leadership relationship as a key factor to achieve educational quality, in light of public policies. When reviewing the regulations of the teaching profession in Colombia, it is concluded that there are missing aspects to achieve that the teacher is really a proactive and empathetic leader. Key factors for teacher training and the improvement of educational quality in the process of thinking about configuring schools as professional learning communities
\end{abstract}

\section{Keywords}

Educational policies; Leadership; teacher professionalization; educational quality; 


\section{Liderança como fator chave para melhorar a profissionalização dos professores e a qualidade educacional}

\section{Resumo}

Este documento aborda algumas das questões conjunturais da profissionalização de professores à luz das políticas públicas na Colômbia e a relação de liderança como um fator-chave na melhoria da qualidade educacional. Também é abordada a relevância das recomendações da OCDE sobre a formação de professores para a Colômbia, por isso é importante realizar uma exploração crítica dos dispositivos de formação de professores à luz das políticas de melhoria, para avançar na análise e desenvolvimento de propostas alternativas e novas diretrizes para o desenvolvimento profissional dos professores. Para o desenvolvimento desta proposta, foi necessário gerar uma ação reflexiva sistemática e crítica sobre a relação de liderança como fator-chave para alcançar a qualidade educacional, à luz das políticas públicas. Ao revisar os regulamentos da profissão docente na Colômbia, conclui-se que faltam aspectos para que o professor seja realmente um líder proativo e empático. Fatores-chave para a formação de professores e a melhoria da qualidade educacional no processo de pensar em configurar as escolas como comunidades de aprendizagem profissional.

\section{Palavras-chave}

Políticas educacionais; Liderança; profissionalização de professores; qualidade educacional; 


\section{Introducción}

Al intentar definir la docencia como una profesión surgen muchas dudas desde una mirada retrospectiva de la actividad docente, y es a partir de la identificación del conjunto de características que se definen particularidades en cada sociedad y en cada nivel del sistema educativo, donde se desempeña esta labor. Desde el punto de vista de Christopher Day, el desarrollo profesional docente consiste en:

todas las experiencias de aprendizaje personal y aquellas actividades conscientes y planificadas que pretenden ser de beneficio directo o indirecto para el individuo, grupo o escuela, que contribuyen, a través de éstas, a la calidad de la educación en el aula. Es el proceso por el cual, solo y con otros, los maestros revisan, renuevan y extienden su compromiso como agentes de cambio hacia los propósitos morales de la enseñanza; y mediante el cual adquieren y desarrollan críticamente conocimientos, habilidades e inteligencia emocional que son esenciales para lograr un buen pensamiento, planificación y práctica profesional con niños, jóvenes y colegas en cada una de las fases de sus vidas docentes (Muijs, Day, Harris \& Lindsay, 2004, p. 295).

Esta definición orienta un plano individual del docente al referirse a todas las experiencias de aprendizaje y otro enfoque más amplio donde se extiende el compromiso docente como agente de cambio, el cual debe tener presente los contextos y es aquí donde las políticas institucionales y el sistema educativo deben hacer la sinergia para lograr una práctica profesional exitosa (Avalos, 2004).

La sociedad continúa entregado a los docentes cada vez más responsabilidad acerca de los resultados del sistema educativo es así como el trabajo docente se torna como una actividad sumamente compleja (Vaillant y Marcelo, 2018), donde la mayoría de los focos están puestos en los resultados dejando de lado, uno de los grandes inconvenientes y al mismo tiempo de difícil solución: el capital cultural de los docentes. Este capital cultural que se construye desde la formación inicial, el servicio y sigue con la actualización permanente de los docentes. En suma, todos los esfuerzos por mejorar significativamente la formación docente, constituyen un avance y un aporte al sistema educativo en general (Avalos, 2000).

Si bien el foco del paradigma educativo se ha ubicado en el aprendizaje del estudiante, de manera que la concepción tradicional del perfil docente como experto académico y mediador dominante del saber no parece ser suficiente para dar respuesta a las 
demandas planteadas (Pavié, 2011), por lo tanto, urge revisar el perfil del docente y redefinir su trabajo y formación permanente para los retos del siglo XXI (Martínez, 2016). Se trata de identificar a los docentes que saben aplicar los conocimientos adquiridos en el periodo de servicio y mostrar su capacidad de liderazgo para crear entornos nuevos que permiten mejorar su aprendizaje y el de sus estudiantes; docentes que saben integrar conocimientos y enfrentarse a la complejidad de formular juicios en situaciones de incertidumbre que con frecuencia estarán acompañados de consecuencias sociales y éticas; docentes que saben comunicar sus conocimientos y razones últimas que sustentan sus acciones no solo ante sus estudiantes sino ante públicos especializados y no especializados de forma clara, sin ambigüedades; docentes que poseen las habilidades de aprendizaje que le permitan continuar estudiando de manera auto dirigida y autónoma a lo largo de su vida profesional (Martínez, 2016; OECD 2015).

Para acercarnos a la situación de la formación docente en Colombia es importante reflexionar de manera sistemática acerca de los logros y desafíos en materia de formación de profesores, así como sobre los avances educativos que intentan garantizar la educación en el marco de las políticas públicas. De esta manera, este documento busca mostrar una reflexión acerca de la profesionalización docente a la luz de las políticas públicas en Colombia y la relación del liderazgo como factor clave para el mejoramiento de la calidad educativa teniendo en cuenta las recomendaciones ultimas de la OECD en materia de educación.

El texto está divido de la siguiente manera: introducción, metodología, desarrollo profesional docente en Colombia, el liderazgo en la formación docente, el liderazgo y la profesión docente desde las políticas educativas y finalmente resultados y conclusión.

\section{Diseño metodológico}

Se realiza un análisis y abstracción de literatura pedagógica y metodológica con la recogida de la información publicada en bases de datos de uso libre y la base de datos del Ministerio de Educación Nacional acerca de los temas relacionados con las políticas públicas de formación docente. Se efectúa en consecuencia, una reflexión sistemática acerca de los logros y desafíos en materia de formación de profesores, así como la relación con aspectos como el liderazgo y la calidad de la educación en Colombia. Se tomaron en cuenta algunas preguntas orientadoras en el desarrollo de este análisis: ¿Se 
identifica el liderazgo docente a partir de las políticas públicas de educación?, ¿Cuál es la relación entre liderazgo y formación docente? Una vez que se estableció una visión general de la investigación sobre el tema, se procedió al análisis para identificar relaciones y enfoque temático de la investigación.

\section{Desarrollo Profesional Docente en Colombia}

En Colombia el Sistema de Formación y Desarrollo Profesional Docente se define como el "conjunto de componentes y actores comprometidos en la ejecución de las políticas de formación docente que impulsan programas, proyectos, estrategias y acciones conducentes al logro de objetivos y metas de mejoramiento de los procesos de formación docente inicial y continua" (MEN, 2012, p, 83).

En cuanto a la regulación de la formación docente en Colombia las normas se definen básicamente hacia la formación docente inicial, con una progresiva regulación nacional o política específica que atienda a la formación continua de docente. La formación en servicio de los docentes es determinada por varios actos normativos, realizados por el Congreso de la República de Colombia y reglamentados por el Ministerio de Educación Nacional, las secretarías de Educación, el Departamento Administrativo de la Función Pública y la Comisión Nacional del Servicio Civil, y rigen principalmente para el sector público (Bautista, 2009). Entre los diferentes actos normativos se definen leyes, decretos, resoluciones, Conpes, entre otros.

Partiendo de la base que considera, que la formación docente también está asociada a la idea de calidad en la educación, de manera general se muestra a continuación los acercamientos en materias de políticas educativas que impulsan, organizan, evalúan y controlan la formación de profesores en Colombia, en orden cronológico:

En 1991 se define la Constitución Política, la cual reguló los diferentes aspectos del Magisterio en el país. Entre los aspectos a destacar de la Constitución tenemos la promulgación de la Ley General de la Educación (Ley 115, 1994).

En 1992 se publica la Ley de Educación Superior (Ley 30 de 1992), la cual determinó que el ejercicio de la docencia es de formación profesional y por lo tanto debe ser universitario. Se define en las instituciones universitarias el concepto de autonomía y por la parte del gobierno el concepto de vigilancia y control

En 1994 a partir de la Ley General de Educación (Ley 115, 1994) se definen los parámetros para la acreditación de las Instituciones Educativas de Educación Superior- 
Facultades de Educación que forman los licenciados, las dos principales instituciones que otorgan títulos para ejercer la docencia. En el artículo 109 del capítulo 2 se declara. (Ley 115, 1994):

La formación de educadores tendrá como fines generales:

a) Formar un educador de la más alta calidad científica y ética;

b) Desarrollar la teoría y la práctica pedagógica como parte fundamental del saber del educador;

c) Fortalecer la investigación en el campo pedagógico y en el saber específico, y

d) Preparar educadores a nivel de pregrado y de postgrado para los diferentes niveles y formas de prestación del servicio educativo.

De acuerdo con la norma anterior, se definen los propósitos o generalidades de la formación de docente, con respecto a la profesionalización, actualización, especialización y perfeccionamiento de los docente, en 1996, a partir Decreto 0709 de abril 17, por el cual se establece el reglamento general para el desarrollo de programas de formación de educadores el cual comprende la formación inicial y de pregrado, la formación de postgrado y la formación permanente o en servicio y además se crean condiciones para su mejoramiento profesional. Con esta norma se estructuran los programas de formación, atendiendo a cuatro campos: formación pedagógica, disciplinaria específica, científica investigativa y deontológica, y valores humanos. Resulta importante resaltar el rol de las Facultades de Educación y de los Departamentos a través de las Secretarías de Educación, Departamentales y Municipales, en el perfeccionamiento y profesionalización de los docentes en ejercicio (Decreto 0709, 1996; MEN, 2016).

Concentrar la mirada en la formación docente para buscar recursos que conduzcan a la calidad, es paralelo a preguntarnos, cómo, en qué y para qué se están formando los docentes, implica mancomunar acciones en un complejo proceso en el que se ven comprometidos sujetos (los mismos docentes), instituciones, administraciones, gobiernos y políticas implicadas con impulsar una apuesta educativa para la mejora escolar. Por lo tanto, estas acciones se deben ver reflejadas en la puesta en marcha de la estructura de la formación de docente, en tres unidades: la formación inicial lo que equivale al pregrado, la formación en servicio o profesionalización y la formación avanzada o estudios de posgrado. Por lo tanto, se destaca desde estos planteamientos la articulación que podría lograrse en la formación docente y la dedicación de 
profesionales docentes que reconocen el saber práctico y teórico de la pedagogía, el fortalecimiento de la investigación y la disposición para servir con alta calidad científica y ética.

De este modo, las propuestas de perfeccionamiento en servicio son diversas y cuentan con nuevos lineamientos comunes para su formulación, algunos de estos se encuentran en diversas partes del "Plan Decenal de Educación 2016-2026" del cual se resalta aspectos que, cabe destacar que sus definiciones aún no se evalúan. Uno de los lineamientos del plan decenal es garantizar en la formación inicial, continua y avanzada de educadores, el enfoque de educación inclusiva, el enfoque de derechos, el uso pedagógico de las TIC (Henao, 2019) y el desarrollo de competencias socioemocionales y ciudadanas, capacidades de liderazgo, para la construcción de paz y equidad; aspectos que de llevar a la práctica reducirían muchos de los inconvenientes de la educación actual en Colombia. Se nota en este documento un paso importante en el lineamiento de los cambios educacionales mostrando un gran interés en el desarrollo profesional docente (PDE 2016-2026, 2017)

Continuando con la normativa en cuanto a la formación de docentes es clave mencionar que a nivel de la educación básica y media está reglamentada bajo dos normas. Los dos estatutos que actualmente acogen a los docentes, El primero es el Decreto 2277 de 1979 y el segundo es el Decreto 1278 de 2002, "El Nuevo Estatuto Docente”. En este nuevo decreto se introduce el concepto profesional de la educación, además de establecer nuevas reglas de formación, ingreso, evaluación, promoción y permanencia en la profesión. Entre ambos decretos se presentan semejanzas y diferencias que han generado un clima particular en el cuerpo docente del sector público e incide en la construcción de una visión frente a su propia labor.

Teniendo en cuenta que la búsqueda de la calidad de la educación en Colombia es uno de los objetivos del Decreto 1278 de 2002 como política pública, no podemos dejar de lado que la educación es la suma de responsabilidades y los esfuerzos del Estado, la familia, los docentes y los mismos estudiantes (Cubillos, 2014). Es por esto que nos enfocamos en analizar la función docente y sus diferencias con la profesionalización del docente.

La profesionalización obedece a la reglamentación, determinación, organización e institucionalización de la profesión; mientras la función docente se acerca más a las acciones útiles de la profesión vislumbradas dentro de la norma, es decir lo definido en el Estatuto Docente, en el cual se imprime la función como: "aquella de carácter 
profesional que implica la realización directa de los procesos sistemáticos de enseñanza - aprendizaje [ ...]" (Decreto 1278, articulo 4; p.1). No obstante, los dos conceptos no pueden quedarse en una definición y es aquí donde entra el sujeto docente con algunas competencias como el liderazgo para humanizar la norma haciendo el aporte desde su rol de agente de cambio social (Cubillos, 2014).

Desde otro punto de vista podríamos decir que la profesionalización es un proceso que depende de la idoneidad en el desempeño del docente y sus competencias demostradas para continuar en la carrera, lo que indica que el ejercicio docente incide en la profesionalización, por lo que ésta de manera intrínseca adquiere un carácter subjetivo, muy propio y partícula de cada docente. Por ahora se puede decir que en Colombia se ha dado un leve progreso en la dirección de políticas articuladas sobre docentes y una muestra de esto ha sido la definición de una serie de normas que apuntan al mejoramiento de la profesionalización docente (Martínez, 2006). Partiendo de las siguientes: subir a nivel universitario la formación inicial docente (1998), definir un registro calificado para los programas de formación docente (Decreto 2566, 2003) reglamentar la calidad y el desarrollo de programas de formación de formadores (Resolución 1036, 2004), norma para Exámenes de Calidad de la Educación Superior y la formulación del Estatuto Docente (Decreto 1278, 2002). Además, La Ley 115 de 1994 a su vez establece que la formación continua comprende actividades de investigación, innovación y actualización. Es muy cierto, que se han dado estos avances acerca de la profesionalización de los docentes, sin embargo, aún falta mucho por discutir desde otras dimensiones como la calidad, es decir que el debate apenas inicia, con puntos pendientes como la discusión sobre los procesos de profesionalización del docente asociados al marcado interés por mejorar la calidad profesional de los docentes, y por ende su calidad de vida. (Bautista, 2009).

\section{El liderazgo en la Profesión Docente}

El liderazgo se puede definir como un conjunto de funciones organizativas en lugar de alguien que ocupa determinadas posiciones formales o administrativas. Más allá de una noción autocrática o jefe que dirige al personal, hoy se habla de nuevos liderazgos, dentro de las interdependencias recíprocas que se dan en cualquier organización (Bolívar, 2014). Luego, el liderazgo educativo, como lo define Robinson \& Timperley (2009) citado por Bolívar (2014) se puede concebir como aquel que dota de un sentido 
común a la institución educativa e influye en el comportamiento de sus actores, teniendo como misión la mejora de la calidad, que se expresa tangiblemente en los aprendizajes de los estudiantes.

[...] concepción de liderazgo destaca su papel en el cambio: liderazgo implica influenciar en la gente para pensar y actuar de forma diferente, ya sea directamente (encuentros cara a cara) o indirectamente (mediante la creación de las condiciones pertinentes). Podemos distinguir entre liderar y gestionar. Gestionar es acerca de mantener operaciones y rutinas; liderazgo trata de recabar apoyos para el posible cambio. Sin embargo, la distinción no se puede establecer radicalmente, pues los gestores precisan de competencias de liderazgo y los líderes necesitan habilidades gestoras (Robinson \& Timperley, 2009, p. 8).

Y es a partir de los aprendizajes de los estudiantes que dicho liderazgo está integrado en las rutinas escolares que están alineadas con los objetivos de mejora, e implica el uso de herramientas inteligentes que están diseñadas para ayudar a los docentes a aprender prácticas pedagógicas más efectivas. Este enfoque de resultados de los estudiantes tiene implicaciones sobre cómo se puede enfocar el liderazgo desde el aula. De acuerdo con algunas investigaciones (Robinson \& Timperley, 2009) son los maestros los que tienen la mayor influencia directa en los estudiantes (Soto, 2014).

El uso del liderazgo en el aula hace parte de la labor del docente y por ende de la formación docente, cada día, de manera que es apenas justo apelar más al ejercicio de liderazgo pedagógico de la dirección escolar, sin embargo, es preciso reconocer las barreras, incrustadas en la cultura escolar, que frenan la práctica, el ejercicio de una dirección pedagógica. Al respecto, algunos de las disyuntivas que marcan las grandes líneas de futuro en la dirección de escuelas, son: liderazgo educativo versus una dirección centrada en la gestión, por un lado; y mayor capacidad para tomar decisiones independientes, con posterior rendimiento de cuentas, versus regulación normativa por la administración educativa (Bolívar, 2014).

De acuerdo con lo anterior, para lograr que las instituciones aprendan y mejoren sus procesos es recomendable pasar del líder formal a un liderazgo colaborativo, donde directivos y profesorado colaboran para resolver los problemas colectivamente, apropiarse la responsabilidad de los éxitos y poniendo los medios para capacitarse en su logro. De manera que liderar se convierte en un esfuerzo sumado por mejorar la calidad de la educación y a su vez la escuela se convierte en una comunidad de aprendizaje para 
la formación parmente de los docentes (Berg \& Zoellick, 2019).). Como un nuevo marco conceptual para analizar y desafiar el liderazgo en la escuela se propone el liderazgo distribuido (Santafé, 2018), el cual es un cambio en la cultura que requiere compromiso e involucra a todos los miembros de la comunidad educativa hacia una mejora académica y gestión escolar. De esta forma el liderazgo distribuido cultiva las habilidades de los otros en una misión común, de tal forma que el liderazgo se desarrolla en todos los niveles (Murillo, 2006).

Si bien estamos en momento de transición de varias dimensiones: políticas, económicas, culturales, tecnológicas y sociales y ante estas el docente por su puesto asume roles ajustados a los contextos para dar cuenta a las necesidades que se le presentan. La profesión docente asume el conjunto de actividades en que los docentes se involucran a lo largo de su carrera, actividades realizadas tanto para mejorar su competencia en la labor, como para lograr unos avances individuales de su profesión. Como resultante de las interacciones se da, un proceso de aprendizaje que tienen lugar en el contexto temporal y espacial de su trabajo y que da lugar a cambios en la práctica docente y en los modos de pensar dicha práctica. A partir de esto podríamos pensar que gran parte de los aprendizajes del docente durante su servicio y su formación permanente, deben estar articulados con las necesidades de desarrollos individuales, institucionales y colectivas en tiempos y espacios compartidos y a lo largo y ancho de la vida de los docentes. Y durante este proceso es cuando se da un acercamiento a la expresión de liderazgo docente.

El liderazgo docente es un término inconsistentemente definido el cual se define a partir de un marco conceptual o una base de investigación relativamente débil (Smylie \& Denny, 1990; York-Barr \& Duke, 2004). Este liderazgo se asocia inicialmente a los procesos de enseñanza y aprendizaje que continuamente mejora el docente, sin embargo, las raíces de acuerdo con algunos estudios (Sebastian, Allensworth \& Huang, 2016), coinciden en que las nociones sobre liderazgo docente se remontan a discusiones sobre la profesionalización docente. En consecuencia, la profesionalización docente puede notarse como la relación entre el liderazgo y la profesión docente, integrado en el lenguaje y la práctica educativa para lograr calidad educativa. 
Algunos gobiernos en la búsqueda de la calidad educativa ponen su lente en el liderazgo docente e impulsan el concepto a través de la profesionalización, aspecto que podría disminuir la cultura aislada de procesos de enseñanza y aprendizaje y mejorar la formación docente, sin embargo, aún queda suelto y algo obsoleto las políticas públicas que deberían actualizarse de acuerdo con las tendencias de la educación (Talbert \& McLaughlin, 1994)

\section{EI liderazgo y la Profesión docente desde las políticas educativas.}

Las investigaciones sobre el liderazgo educativo, han hecho visible la debilidad de la mirada del liderazgo como un fenómeno individual, y jerárquico. En consecuencia, se ha dado un giro en materia de investigación sobre liderazgo educativo hacia los estilos y el rol de liderazgo del director, como actor principal que jalona un proceso (Cruz-Gonzalez, Segovia, \& Rodríguez, 2019). Por lo tanto, la tendencia es hacia perspectivas mancomunadas, y focalizadas en el conocimiento sobre prácticas de liderazgo de los diferentes actores que participan en las decisiones de formación, desarrollo e innovación escolar (Maureira, Moforte, \& González, 2014).

Desde la teoría del Liderazgo distribuido lograr un docente líder puede hacer parte de la misma formación permanente que se realiza en las instituciones educativas y puede ser un propósito implícito de las directivas al buscar la creación de comunidades de aprendizaje, comunidades de prácticas, grupos colaborativos, entre otras estrategias que abonarían el terreno para lograr más liderazgo entre los docentes.

Por otra parte, entre las recomendaciones que hace la OECD (Radinger, Echazarra, Guerrero y Valenzuela, 2018), a Colombia, es la de promover el desarrollo de una nueva visión del profesionalismo docente basada en el compromiso efectivo y el consenso con las partes interesadas. Es así como se hace necesario avanzar hacia un modelo más integral de profesionalismo que considere las formas en que la enseñanza y el aprendizaje se integran en sistemas complejos y contextos comunitarios de manera que adoptar los enfoques del liderazgo en un gana-gana desde los puntos de vista gubernamentales, institucionales e individuales.

Una nueva visión del profesionalismo docente debería promover la colaboración entre los docentes para apoyar el aprendizaje y el desarrollo de los estudiantes con un 
claro apoyo de la dirección escolar. La calidad de los docentes individuales es fundamental, pero también lo es el entorno en el que trabajan los docentes.

Lo claro es que hoy en Colombia la profesión docente no es atractiva para los estudiantes altamente calificados, lo que incide negativamente en la calidad del sistema educativo. Para tomar acción en este aspecto Colombia deberá proponer iniciativas para alentar a los estudiantes con mayor rendimiento y mejor cualificados a que ingresen en la carrera docente y disuadir así a aquellos que no cuentan con las competencias ideales. Adicionalmente se resalta que se han realizado avances importantes en la profesionalización docente, comenzando por el decreto 1278 de 2002 y las iniciativas de formación a nivel central; pero aún hay retos en la implementación del nuevo estatuto, en una nueva visión de la profesión y en mejorar la formación inicial y en servicio.

\section{Resultados}

El liderazgo docente continúa cobrando importancia y por su parte los docentes esta tomando un rol mucho mas activo en lo que se refiere a procesos de enseñanza y aprendizaje frente a las diferentes situaciones que se presentan en el aula, independiente de politicas de educación y normas que aún no se actualizan. Como resultados y teniendo en cuenta la revisión anterior se consideran ciertos aspectos que continúan en la discusión:

- Promover una comprensión común y basada en evidencia de la práctica docente efectiva;

- Mejorar las evaluaciones formativas de los maestros en las escuelas;

- Crear oportunidades para que los docentes asuman otras tareas y roles de liderazgo en línea con una nueva organización de escuelas;

- Utilizar recursos para la remuneración de los docentes para igualar las condiciones de trabajo de los docentes del nuevo estatuto y mejorar el sistema actual de progresión profesional;

- Las competencias docentes frente a las nuevas tecnologías.

- Establecer una sólida base de conocimientos sobre el uso de su tiempo por parte de los docentes.

Entre las recomendaciones entregadas a Colombia por la OECD (Radinger, Echazarra, Guerrero y Valenzuela, 2018), se hace énfasis en la necesidad de establecer “oportunidades de formación, evaluaciones eficaces y estructuras adecuadas de remuneración y progresión profesional”, en la profesión de docente. Lo cual se debe en 
gran parte a las exigencias de programas de formación docente los cuales son muy débiles y no se lea ha dado la atención adecuada en los últimos 25 años. En esta misma línea la participación de profesores y directivos en actividades de desarrollo profesional para actualizar conocimientos y asumir nuevas funciones, es muy baja. Con esto se evidencia una vez la necesidad de fortalecer el liderazgo de los docentes y directivos docentes en la construcción de nuevas políticas educativas que enriquezcan los perfiles de docentes y la búsqueda de estrategias que mejoren su formación docente permanente.

Además, algunas investigaciones surgieren tener muy presente las influencias externas e internas en la construcción de la identidad profesional de los directores de las escuelas.

Después de revisar la normatividad de la profesión docente en Colombia, conocer algunas de las recomendaciones que se hacen desde organismos nacionales e internacionales y acercarse a la realidad escolar, podemos decir que es mucho lo que falta por hacer para logar que los docentes asuman roles de liderazgos y que sea esto, un factor clave para la formación docente y el mejoramiento de la calidad educativa.

\section{Conclusiones}

En consecuencia, desde otras perspectivas e investigaciones consultadas se puede apreciar que podemos lograr la ruta para caminar hacia la calidad educativa y ya tenemos algunos avances como los siguientes:

Las discusiones en torno a la profesionalización docente. Con la recomendación de la OECD (Radinger, Echazarra, Guerrero y Valenzuela, 2018), para promover una nueva visión del profesionalismo docente y hacer que la asignación de docentes sea más eficiente y equitativa. Es claro que se han tomado medidas considerables para profesionalizar la enseñanza en las últimas dos décadas. En general, las reformas pasadas se han centrado principalmente en el docente individual y han prestado menos atención a las condiciones organizativas e institucionales requeridas para la enseñanza y el aprendizaje efectivos. La mejora escolar sostenible, sin embargo, es un proceso complejo que necesita tiempo, liderazgo educativo y un sentido de responsabilidad colectiva.

La sugerencia frente a este aspecto es que Colombia debería desarrollar un modelo más completo de profesionalismo docente. Es decir que si bien existe un estatuto (1278 
de 2002) será bueno trabajar en una actualización que incluya aspectos con una visión de las escuelas como comunidades profesionales y considerar las necesidades de los maestros rurales, en particular para su aprendizaje y desarrollo. Tener presente entre las consideraciones que la contratación de docentes en Colombia se basa esencialmente en los derechos de los docentes más que en las necesidades de los estudiantes, lo que genera ineficiencias e inequidades en la asignación de docentes. Lo anterior sugiere tener presente las necesidades colectivas de los estudiantes y por su puesto hacer partícipes a los mismos docentes para la actualización de nuevas normas.

Las instituciones de educación por su parte y apoyándose en sus conceptos de autonomía deberán pensar en configurar las escuelas como comunidades profesionales de aprendizaje, es decir como una organización que aprende y cualifica a los que trabajan en ella. Según algunos autores (Krichesky y Murillo 2011), "la Comunidad Profesional de Aprendizaje" es, de hecho, una comunidad de líderes, donde el liderazgo docente es la base para promover y sostener esta estrategia de mejora, cuando todos los miembros de la comunidad educativa se sientan empoderados y comprometidos con los procesos de cambio y mejora en la institución educativa. Donde el liderazgo docente sea la base para promover y sostener esta estrategia de mejora continua y de transformación de las instituciones educativas.

Finalmente, el desarrollo profesional docente se puede potenciar en la medida en que las instituciones de educación desarrollen la capacidad para organizarse como una comunidad profesional de aprendizaje. 


\section{Referencia}

Avalos, B. (2000). El desarrollo profesional de los docentes. Proyectando desde el presente al futuro. Documento de trabajo presentado al VII Seminario sobre prospectivas de la educación en la región de América Latina y el Caribe, organizado por la Oficina Regional de Educación de la Unesco. Santiago de Chile, 23, 25.

Avalos, B. (2004). CPD policies and practices in the Latin American region. International handbook on the continuing professional development of teachers, 119145.

Bautista M. (2009). La profesionalización docente en Colombia. Revista colombiana de sociología, $\quad 32(2), \quad$ 111-132. Consultado en https://revistas.unal.edu.co/index.php/recs/article/viewFile/12705/13316

Berg, J., \& Zoellick, B. (2019). Teacher leadership: toward a new conceptual framework. Journal of Professional Capital and Community. Vol. 4 No. 1, pp. 2-14. https://doi.org/10.1108/JPCC-06-2018-0017

Bolívar, A. (2014). Liderazgo educativo y desarrollo profesional docente: Una revisión internacional. En J. Ulloa y S. Rodriguez (eds): Liderazgo escolar y desarrollo profesional de docentes: Aportes para la mejora de la escuela. Universidad de Concepción (Chile). RIL editores, pp. 61-103.

Congreso de la Republica de Colombia (8 de febrero de 1994) Ley General de Educación. [Ley 115 de 1994]. Recuperado de https://www.mineducacion.gov.co/1621/articles-85906_archivo_pdf.pdf

Congreso de la Republica de Colombia (30 de diciembre de 1992) Ley 30 de la Educación Superior. [Ley 30 de 1992]. Recuperado de https://www.cna.gov.co/1741/articles-186370_ley_3092.pdf

Cubillos, C. (2014). Impacto del Nuevo Estatuto de Profesionalización en la función docente en Colombia. Análisis de los dos estatutos vigentes: Decreto 2277 de 1979 y Decreto 1278 del 2002. Revista Colombiana de Sociología, 37(2), 213250.

Cruz-Gonzalez, C., Segovia, J., \& Rodríguez, C. (2019). School principals and leadership identity: A thematic exploration of the literature. Educational Research, 61(3), 319-336. https://doi.org/10.1080/00131881.2019.1633941

Flores, A. (1994). Liderazgo en acción. México: Grijalva.

Henao, J. (2019). Pedagogías y estrategias para la compresión lectora en el escenario de las TIC. CEDOTIC, 4 (2), pp. 1-4. Recuperado de http://investigaciones.uniatlantico.edu.co/revistas/index.php/CEDOTIC/article/vi ew/2379 
Krichesky, G. y Murillo, F., (2011). Las Comunidades Profesionales de Aprendizaje. una estrategia de mejora para una nueva concepción de escuela. REICE. Revista Iberoamericana sobre Calidad, Eficacia y Cambio en Educación, 9 (1), 65-83.

Martínez, M. (2016). La formación inicial de los maestros: una responsabilidad compartida. Bordón. Revista de pedagogía, 68(2), 9-16.

Martínez, L. A. (2006). El caso de Colombia. En D. Vaillant y C. Rossel (Eds.), Maestros de Escuelas Básicas en América Latina. Hacia una Radiografía de la Profesión. Santiago: PREAL. Consultado de http://iwww.thedialogue.org/PublicationFiles/Maestrosbasicas.pdf

Murillo, F. (2006). Una dirección escolar para el cambio: del liderazgo transformacional al liderazgo distribuido. REICE: Revista Electrónica Iberoamericana sobre calidad, eficacia y cambio en educación.

Muijs, D., Day, C., Harris, A., \& Lindsay, G. (2004). 12 Evaluating CPD: an overview. International handbook on the continuing professional development of teachers, 291.

MEN. (2012). Políticas y sistema colombiano de formación y desarrollo profesional docente. Recuperado de: https://www.mineducacion.gov.co/cvn/1665/articles208603_archivo_pdf.pdf

MEN (2016). Manual de Funciones, Requisitos y Competencias para Directivos Docentes y Docentes. Aprobado mediante Resolución 15683. Bogotá, DC. Ministerio de Educación Nacional.

OECD (2015). Educación y equidad por una economía más justa. En OCDE, Colombia: políticas prioritarias para un desarrollo inclusivo. Serie Mejores Políticas. Disponible en https://www.oecd.org/about/publishing/colombia-politicasprioritarias-para-un-desarrollo-inclusivo.pdf

Pavié, A. (2011). Formación docente: hacia una definición del concepto de competencia profesional docente. REIFOP, 14 (1), 67-80. Recuperado de https://www.aufop.com/aufop/revistas/arta/digital/158/1620

Plan Nacional Decenal de Educación 2016 2026. (2017). El camino hacia la calidad y la equidad. http://www.plandecenal.edu.co/cms/images/PLAN\%20NACIONAL\%20DECE NAL\%20DE\%20EDUCACION\%202DA\%20EDICION_271117.pdf

Presidencia de Republica de Colombia (abril 17 de 1996) Decreto 0709 de abril 17 de 1996. [Decreto 0709 de 1996]. Recuperado de https://www.mineducacion.gov.co/1621/articles-86215 archivo pdf.pdf

Presidencia de Republica de Colombia (septiembre 14 de 1979) Decreto 2277 de septiembre 14 de 1979. [Decreto 2277 de 1979]. Recuperado de https://www.mineducacion.gov.co/1621/articles-103879_archivo_pdf.pdf 
Presidencia de Republica de Colombia (junio 19 de 2002) Decreto 1278 de junio 19 de 2002. [Decreto 1278 de 2002]. Recuperado de https://www.mineducacion.gov.co/1621/articles-86102_archivo_pdf.pdf

Radinger, T., Echazarra, A., Guerrero, G. \& Valenzuela, J. (2018), OECD Reviews of School Resources: Colombia 2018, OECD Reviews of School Resources. Paris: OECD Publishing https://doi.org/10.1787/9789264303751-en

Robinson, V., \& Timperley, H., (2007). The leadership of the improvement teaching and learning: Lessons from initiatives with positive outcomes for students. Australian Journal of Education, 51(3), 247-262. https://doi.org/10.1177/000494410705100303

Santafé, A. (2017). Hacia una propuesta de liderazgo Educativo y Eduentretenimiento. Cedotic, Vol.2 Núm. 2.

Sebastian, J., Allensworth, E., \& Huang, H. (2016). The role of teacher leadership in how principals influence classroom instruction and student learning. American Journal of Education, 123(1), 69-108. http://doi/pdfplus/10.1086/688169

Smylie, M. A., \& Denny, J. W. (1990). Teacher leadership: Tensions and ambiguities in organizational perspective. Educational administration quarterly, 26(3), 235259. https://doi.org/10.1177/0013161X90026003003

Soto-Molina, Jairo E. (2014). La Ética de la Investigación en las Ciencias Humanas o Sociales. Amauta, 12(23).

Talbert, J. E., \& McLaughlin, M. W. (1994). Teacher professionalism in local school contexts. American journal of education, 102(2), 123-153.

Velázquez Valadez, G. (2005). Liderazgo empático: un modelo de liderazgo para las organizaciones. Mundo siglo XXI, (1), 71-86.

Vezub, L. (2005). Tendencias internacionales de desarrollo profesional docente. La experiencia de México, Colombia, Estados Unidos y España. Ciencia y Tecnología de Argentina.< http://www. preal. org/BusquedaNN. asp> [Consulta: 27 junio 2009].

Vaillant, D. y Marcelo, C. (2018). Hacia una formación disruptiva de docentes, Madrid: Narcea

York-Barr, J., \& Duke, K. (2004). What do we know about teacher leadership? Findings from two decades of scholarship. Review of educational research, 74(3), 255316. https://doi.org/10.3102/00346543074003255 